\title{
Uso Racional dos Cabos-Eletrodos na Estimulação Cardíaca Artificial
}

\author{
Rational Use of Leads in Artificial Cardiac Pacing \\ Carlos Eduardo Duarte ${ }^{1, *}$, André Brambilla Sbaraini ${ }^{2}$
}

ORCID IDs

Duarte CE (D) https://orcid.org/0000-0001-6671-0820

Sbaraini AB (D) https://orcid.org/0000-0002-5193-0860

\begin{abstract}
RESUMO
Introdução: Os dispositivos cardíacos eletrônicos implantáveis (DCEls) são terapia consagrada para o tratamento de bradiarritmias, prevenção de morte súbita ou insuficiência cardíaca. Desde o primeiro implante de marcapasso transvenoso há mais de 60 anos, ocorreram avanços tecnológicos dos dispositivos e melhorias nas técnicas cirúrgicas. No entanto esse tipo de terapia ainda está associado a complicações significativas, a maioria relacionada ao implante dos cabos-eletrodos transvenosos. Objetivo: apresentar uma reflexão sobre como praticar o uso racional do implante de cabos-eletrodos e propor estratégias e alternativas para postergá-lo ou evitá-lo, com base nos conhecimentos atuais nos diversos campos da estimulação cardíaca artificial. Métodos: Revisão da literatura que utilizou artigos de 1995 a 2019, de diversas plataformas e revistas. Conclusão: Há a expectativa de que nos próximos anos ocorram avanços tecnológicos e de conhecimento no campo da estimulação leadless, permitindo que esses dispositivos sejam incorporados na prática clínica de maneira rotineira. Atualmente, se o implante de eletrodos ventriculares nos casos de doença do nó sinusal com condução atrioventricular preservada for racionalizado, o implante de eletrodos atriais nos cardiodesfibriladores implantáveis (CDI) sem necessidade de estimulação antibradicardia ou dos eletrodos ventriculares nos casos sem a necessidade de estimulação antitaquicardia (ATP) considerando o implante de CDIs subcutâneos, este artigo terá cumprido o seu papel.
\end{abstract}

PALAVRAS-CHAVE: Marcapasso artificial; Síndrome do nó sinusal; Morte súbita cardíaca; Insuficiência cardíaca.

\begin{abstract}
Introduction: Cardiovascular implantable electronic device (CIEDS) are a proven therapy for the treatment of bradyarrhythmias, prevention of sudden death or heart failure. Since the first transvenous pacemaker implantation more than 60 years ago, technological advances in devices and improvements in surgical techniques have occurred. However, this type of therapy is still associated with significant complications, most of them related to the implantation of transvenous leads. Objective: To present a reflection on how to practice the rational use of lead implantation and propose strategies and alternatives to delay or avoid it, based on the current knowledge in the various fields of artificial cardiac stimulation. Methods: Review of literature that used articles from 1995 to 2019, from several platforms and periodicals. Conclusion: There is an expectation that in the coming years there will be technological and knowledge advances in the field of leadless stimulation, allowing these devices to be incorporated into clinical practice in a routine manner. Currently, if the implantation of ventricular electrodes in cases of sinus node disease with preserved atrioventricular conduction is rationalized, the implantation of atrial electrodes in implantable cardioverter-defibrillators (ICD) without the necessity of antibradicardia stimulation or ventricular electrodes in cases without the necessity of antitachycardia stimulation (ATP) considering the subcutaneous ICD implantation, this article will have fulfilled its role.
\end{abstract}

KEYWORDS: Artificial pacemaker; Sinus node syndrome; sudden cardiac death; Heart failure.

1.Centro Avançado de Ritmologia e Eletrofisiologia - São Paulo (SP), Brasil.

2.Hospital Beneficência Portuguesa de São Paulo - São Paulo (SP), Brasil.

Recebido: Fev. 17, 2020 | Aceito: Mar. 30, 2020

*Autor correspondente: carlosduarte@ritmologiacare.com.br

Editor de seção: José Tarciso Medeiros de Vasconcelos 


\section{INTRODUÇÃO}

Os dispositivos cardíacos eletrônicos implantáveis (DCEIs) são terapia consagrada para o tratamento das bradiarritmias, prevenção de morte súbita ou insuficiência cardíaca. O número de implantes de DCEIs assim como o número de cabos-eletrodos $(\mathrm{CE})$ por dispositivo tem crescido nos últimos anos ${ }^{1,2}$ e estima-se que anualmente são implantados cerca de 1 milhão de dispositivos ao redor do mundo ${ }^{3}$.

Desde o primeiro implante de marcapasso transvenoso há mais de 60 anos ocorreram avanços tecnológicos dos dispositivos e melhorias nas técnicas cirúrgicas. No entanto esse tipo de terapia ainda está associado a complicações significativas, a maioria relacionada ao implante dos caboseletrodos transvenosos: desde o acesso venoso, local de implante, até problemas estruturais levando aos principais recalls da indústria ${ }^{4-7}$.

Há relatos de taxas de até $12 \%$ de complicações em curto prazo relacionadas a técnica cirúrgica em alguns centros ${ }^{8,9}$, que incluem pneumotórax, tamponamento cardíaco, hematoma de loja e deslocamento de eletrodos ${ }^{10-12}$.

Os cabos-eletrodos são a maior fonte de problemas do sistema, podem causar complicações como obstrução venosa, regurgitação tricúspide, perfurações e ser fonte de endocardite infecciosa ${ }^{13,14}$, podendo essa última ter uma taxa de mortalidade de 12 a $31 \%{ }^{15,16}$, acarretando longo tempo de permanência hospitalar e altos custos aos sistemas de saúde ${ }^{17}$.

A longo prazo, os cabos-eletrodos certamente apresentarão complicações estruturais como: lesão do isolante, fratura do condutor ou aumentos proibitivos de impedância, que podem colocar o paciente em risco ou expô-lo a intervenções de extração que podem estar associadas a uma morbimortalidade significativa ${ }^{18,19}$.Frente a esse cenário, as alternativas para se minimizar o número de eletrodos implantados por paciente são necessárias.

\section{OBJETIVO}

O presente texto busca apresentar uma reflexão sobre como praticar o uso racional do implante de cabos-eletrodos, assim como propõe estratégias e alternativas para postergá-lo ou evitá-lo, com base nos conhecimentos atuais nos diversos campos da estimulação cardíaca artificial.

\section{CENÁRIOS}

\section{Doença do nó sinusal}

Pacientes com doença do nó sinusal (DNS) podem ser tratados com marcapassos monocamerais atrial (AAI) ou ventricular (VVI), ou bicamerais (DDD). Tanto o marcapasso AAI como o DDD preservam o sincronismo entre a contração atrial e a ventricular, fazendo com que o número de implantes de marcapassos monocamerais ventriculares caísse vertiginosamente ao longo do tempo.

Apesar de não ter sido observado ganho na sobrevida com a estimulação fisiológica AAI ou DDD em comparação à ventricular exclusiva (VVI), há grande vantagem nesse modo de estimulação por apresentar menores taxas de fibrilação atrial e síndrome do marcapasso. Após a descoberta do prejuízo na função ventricular ocasionado por estimulação desnecessária do ventrículo direito, as empresas investiram no desenvolvimento de algoritmos que promovessem a mínima estimulação ventricular e aparentemente resolvessem a questão ${ }^{20-23}$. Portanto o peso da decisão do médico sobre o tipo de dispositivo a ser implantado diminuiu e o implante de aparelhos de dupla-câmara com possibilidade de somente estimular o ventrículo em casos de necessidade se tornou a conduta padrão. Passaram-se a utilizar marcapassos de duplacâmara sofisticados para prevenir uma possível necessidade evolutiva da doença do nó sinusal associada à doença do nó atrioventricular.

Estudo histórico ${ }^{24}$ de 2005 comparando os sistemas monocamerais e bicamerais nesse cenário demonstrou que a estimulação em modo DDD estava associada a maiores taxas de fibrilação atrial em comparação à estimulação atrial exclusiva (AAI) e que as complicações cirúrgicas foram mais frequentes em implantes de cabos-eletrodos ventriculares, chamando a atenção da melhor relação custo-efetividade da estimulação atrial na DNS.

Os modos de estimulação baseados no átrio (AAIR e DDDR) com gerenciamento da estimulação ventricular por algoritmos com mudanças de modo ou histerese AV foram comparados no estudo DANPACE, que demonstrou que ambos apresentaram taxas de mortalidade semelhantes e revelou o dobro da taxa de reintervenções com a estimulação AAIR. As reoperações ocorreram, na maioria dos casos, por necessidade de upgrade de AAIR para DDDR, consequente ao desenvolvimento de bloqueio atrioventricular, o que pode 
sugerir alguma vantagem em se implantar o sistema bicameral. Naqueles com condução atrioventricular preservada, maioria dos portadores de doença do nó sinusal, os algoritmos que evitem a estimulação ventricular desnecessária foram amplamente recomendados conforme mencionado anteriormente. A mensagem na época foi de que até $18 \%$ dos pacientes arrolados para o braço AAIR foram convertidos a algum tipo de estimulação ventricular (VVI ou DDD). No entanto, sob a visão da racionalização do uso dos cabos-eletrodos, $82 \%$ foram implantados desnecessariamente já no primeiro procedimento ${ }^{25}$.

Em um estudo mais recente ${ }^{26}$, a segurança e eficácia da estimulação em modo AAIR na DNS foram avaliadas em pacientes selecionados. Entre os 85 pacientes remanescentes no estudo acompanhados por um período médio de 10,6 \pm 0,6 anos, $78(91,8 \%)$ não necessitaram de implante de eletrodo ventricular.

No final do seguimento, 31 pacientes $(39,7 \%)$ estavam vivos (seguimento médio $14,3 \pm 0,7$ anos), enquanto 47 pacientes $(60,3 \%)$ foram a óbito (seguimento médio $8,1 \pm 0,7$ anos). Não houve mortes cardíacas súbitas que pudessem ser atribuíveis ao bloqueio $\mathrm{AV}$.

Durante o curso do estudo, 7 (8,2\%) pacientes foram submetidos a implante de eletrodo ventricular (desfecho primário) pelos seguintes motivos: 2 pacientes apresentaram pré-síncope/síncope por bloqueio AV; 1 paciente apresentou deslocamento recorrente do eletrodo atrial; 3 pacientes desenvolveram fibrilação atrial sintomática com necessidade de implante de eletrodo ventricular devido à baixa resposta ventricular consequente ao uso de betabloqueadores; 1 paciente foi submetido a upgrade eletivo para sistema de estimulação
DDD, na ocasião da troca do gerador, por apresentar bloqueio de Wenckebach a taxas de estimulação inferiores a 120 bpm. $\mathrm{O}$ implante desses eletrodos transcorreu sem intercorrências. $\mathrm{O}$ tempo médio para upgrade foi de 5,9 anos e a necessidade de adicionar um eletrodo ventricular ocorreu na taxa de 7,8 por 1000 marcapassos/ano. Durante o período de seguimento, 30 pacientes $(33,7 \%)$ foram submetidos à troca de gerador mantendo o marcapasso AAIR.

Os resultados deste estudo foram muito semelhantes ao estudo DANPACE, porém os autores concluíram que a estimulação AAIR deve ser considerada em pacientes selecionados com DNS sem bloqueio $\mathrm{AV}$, uma vez que em 91,8\% dos pacientes esse modo de estimulação está associado a custos mais baixos, menos eletrodos e menos estimulação ventricular direita; principalmente pelo fato desses marcapassos estarem sendo implantados em uma população cada vez mais idosa, com expectativa de vida limitada devido a outras comorbidades ${ }^{26}$. Nesse caso, mais de $90 \%$ de eletrodos ventriculares poderiam ser evitados ou postergados.

A capacidade de refletir sobre o assunto foi sendo aos poucos ceifada a ponto de não haver nas últimas Diretrizes Brasileiras de Dispositivos Cardíacos Eletrônicos Implantáveis ${ }^{27}$ orientação específica sobre o tipo de dispositivo a ser implantado, o que não difere muito das diretrizes europeias $^{28}$, que citam o marcapasso monocameral atrial (AAI), mas como segunda ou terceira opção desde que se considere a utilização dos algoritmos de mínima estimulação ventricular (AVM) nos dispositivos bicamerais, conforme ilustrado na Fig. 1.

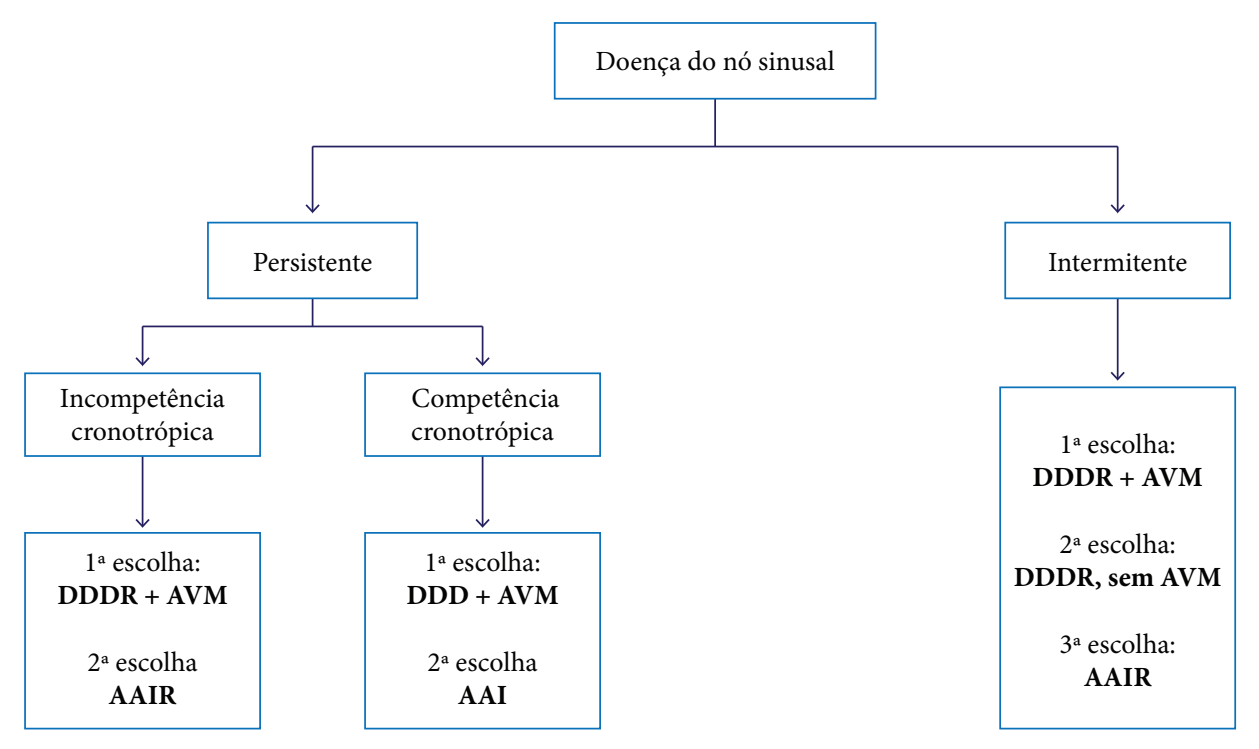

Figura 1. Fluxograma com as recomendações das últimas Diretrizes Europeias sobre o modo de estimulação na DNS²8. 
Em contraponto, o marcapasso monocameral tem destaque nas Diretrizes de 2018 das sociedades americanas ${ }^{29}$ que estudam os distúrbios de condução: seja ele VVI nos casos em que se visa apenas segurança em pacientes com baixa probabilidade de estimulação (classe IIa), ou como AAI (classe I) na DNS com condução AV preservada, conforme a Fig. 2.

A discussão da utilização de marcapassos monocamerais (principalmente AAI) na DNS deve ser retomada. Considerando-se as variáveis como taxa de complicações, custo do procedimento, exposição a lesões vasculares como tromboses e futura necessidade de extração, assim como baixa taxa de associação à doença do nó atrioventricular, a abordagem desse tema está mais que justificada. $\mathrm{O}$ mais preocupante é a cultura da automatização do pensamento de que um cabo-eletrodo a mais "não traz nenhuma consequência" principalmente naqueles mais de $90 \%$ que nunca precisarão deles.

\section{Prevenção de morte súbita}

O cardiodesfibrilador implantável (CDI) é o dispositivo de escolha para o manejo de arritmias ventriculares e prevenção de morte súbita. $\mathrm{O}$ seu benefício é bem estabelecido, porém está sujeito às mesmas complicações que podem ocorrer nos DCEIs mencionadas anteriormente, com o agravante de apresentar maiores taxas de infecção comparado aos marcapassos transvenosos convencionai ${ }^{30}$, além de já ter sido demonstrado por um estudo que mais de $20 \%$ dos pacientes apresentarão algum tipo de disfunção nos eletrodos dentro de 10 anos $^{31}$. Portanto a discussão do implante de eletrodos desnecessários nesse cenário se torna mais importante do que na doença do nó sinusal. No entanto as indicações para implante de CDI na prevenção primária e secundária são definidas nas diretrizes brasileiras e internacionais sem quaisquer recomendações específicas sobre a escolha entre implante de CDI monocameral ou bicameral.

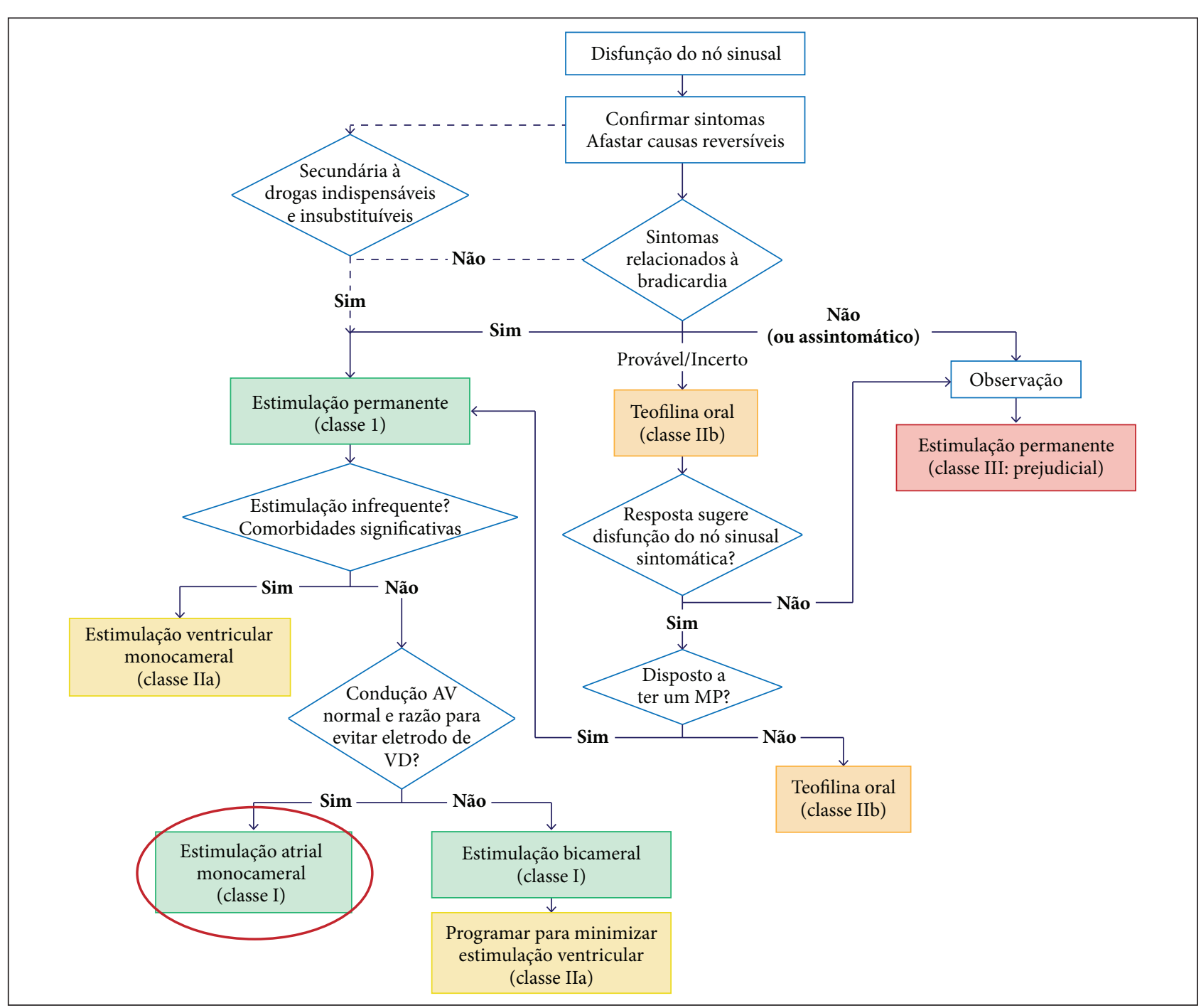

Figura 2. Fluxograma com as recomendações das Sociedades Americanas para implante de marcapasso na DNS 29 . 
Em comparação com o CDI monocameral (CDI-VVI), o CDI bicameral (CDI-DDD) está associado à maior taxa de complicações periprocedimento e mortalidade intrahospitalar ${ }^{32}$, sendo sua indicação questionável a pacientes que não necessitem de estimulação cardíaca para bradiarritmias.

Para evitar complicações relacionadas aos eletrodos transvenosos, atualmente dispõe-se do CDI subcutâneo. Mas no Brasil é preciso evoluir nessa discussão, pois há indícios de que são implantados em média 84-90\% de CDIs bicamerais em detrimento de CDIs monocamerais segundo dados fornecidos pelos fornecedores.

\section{Cardiodesfibrilador implantável (CDI) Dupla-câmara ou monocâmara}

Os pacientes com indicação de CDI e estimulação para bradicardia e/ou terapia de ressincronização cardíaca (TRC) terão indicação de implante de CDI transvenoso (DDD ou VVI).

As indicações para implante de CDI na prevenção primária e secundária são definidas nas diretrizes brasileiras e internacionais. Porém não há recomendações específicas sobre a escolha entre implante de CDI-VVI ou CDI-DDD.

Uma coorte longitudinal retrospectiva ${ }^{32}$ foi realizada para determinar a taxa de complicações intra-hospitalares entre 104.049 pacientes que receberam CDI DDD e VVI, entre 1 de janeiro de 2006 e 31 de dezembro de 2007, em diversos centros.

Os dispositivos bicamerais foram implantados em 64.489 pacientes (62\%). Os efeitos adversos foram mais frequentes nos implantes de dispositivos dupla-câmara comparados aos de monocâmara (3,17\% vs. $2,11 \%, \mathrm{p}<0,001)$, assim como a taxa de mortalidade intra-hospitalar $(0,40 \%$ vs. $0,23 \%, \mathrm{p}<0,001)$. Após o ajuste para dados demográficos, comorbidades médicas, dados de testes de diagnóstico e indicação do CDI, as chances de qualquer complicação (razão de possibilidades: 1,40; intervalo de confiança de 95\%: 1,28 a 1,52; p <0,001) e mortalidade hospitalar (razão de possibilidades: 1,45; Intervalo de confiança de 95\%: 1,20 a 1,$74 ; p<0,001)$ foram aumentados com o implante de CDI-DDD em relação ao CDI-VVI ${ }^{32}$.

Em um registro israelense ${ }^{33}$ publicado em 2016 que incluiu um total de 1125 pacientes, foram comparados os desfechos clínicos (mortalidade, admissões hospitalares por insuficiência cardíaca [IC], e terapia do CDI) entre implante de CDI-VVI × CDI-DDD para prevenção primária de morte súbita. Desses pacientes, 37\% receberam CDI-VVI e 63\% CDI-DDD, o tempo médio de seguimento foi de 22 meses, a fração de ejeção média foi de 30\% e a média de duração do QRS foi de 103ms nos dois grupos. Não houve diferença significativa na taxa de mortalidade, admissões por IC, terapia apropriada ou inapropriada, ou no tempo para atingir qualquer um dos desfechos. Usando análise multivariada, o CDI-VVI não foi associado a maior risco de morte ou admissão por IC. Em um subgrupo de pacientes com miocardiopatia isquêmica, o dispositivo monocameral esteve associado a uma maior taxa de terapia inapropriada. Os autores concluíram que mais estudos prospectivos seriam necessários para avaliar o benefício do CDI-DDD na redução das taxas de terapia inapropriada ${ }^{33}$.

Mais recentemente foi publicado um estudo ${ }^{34}$ que incluiu um total de 2240 pacientes submetidos a implante de CDI em 45 centros alemães, entre janeiro de 2007 e março de 2011, sendo realizada análise relativa às características dos pacientes, dados dos procedimentos e complicações, em período de seguimento de 1 ano, comparando o CDI-VVI com CDI-DDD. Desses pacientes, 1629 foram submetidos a implante de CDI-VVI e 611 DDD; no grupo VVI, foram 1358 indivíduos do sexo masculino, com FE $=34 \% \pm 13 \%$; no grupo DDD, 491 do sexo masculino, com FE $=35 \% \pm 14 \%$. Os pacientes do grupo DDD eram significativamente mais velhos ( $66 \pm 12$ vs. $63 \pm 13$; $p<0,001$ ); o histórico de fibrilação atrial e implante para prevenção primária foi menor neste grupo. Os distúrbios de condução AV e BRE foram mais frequentes no grupo DDD. O número de complicações intrahospitalares foi significativamente maior no grupo DDD ( $3,0 \%$ vs. $1,2 \% ; p=0,003 / n=27$ de 604 pacientes vs. 41 de 1623 pacientes). Além disso, maior taxa de mortalidade foi observada em pacientes com sistema de CDI-DDD (1,0\% vs. $0,0 \% \mathrm{p}<0,001 ; \mathrm{n}=6$ de 611 pacientes vs. 0 de 1629 pacientes). Após 1 ano de acompanhamento, os pacientes submetidos ao implante de CDI-DDD apresentaram incidência aumentada de revisões do dispositivo, re-hospitalização e mortalidade, sem atingir significância estatística. Este estudo demonstrou que, na ausência de significativa doença do nó sinusal ou atrioventricular, dispositivos dupla-câmara estão associados a maior taxa de complicações periprocedimento assim como maior mortalidade, sendo o benefício da sua indicação incerto para pacientes que não possuem tais distúrbios. Portanto os autores concluem que, na ausência de bradicardia relevante (DNS ou BAV), o implante de CDI-DDD não é justificado ${ }^{34}$. 
O implante de CDI dupla-câmara se faz necessário se, no momento do procedimento, o paciente apresentar também indicação de implante de marcapasso. Não se justifica o implante de um eletrodo a mais simplesmente para utilizá-lo como discriminador das taquicardias. Não houve redução de choques inapropriados nos dispositivos bicamerais $^{35,36}$. No entanto sabe-se que a visualização dos eletrogramas atriais torna a interpretação do traçado mais precisa (Fig. 3) ${ }^{37}$.

Uma das empresas do mercado (Biotronik) possui dispositivos da linha DX que se preocupa com a manutenção dos eletrogramas e discriminadores atriais com implante de apenas um eletrodo. Diferentemente dos sistemas VDD tradicionais (eletrodo único com estimulação ventricular e sensibilidade atrioventricular), o CDI DX utiliza um dipolo atrial com espaçamento de $15 \mathrm{~mm}$ e com sistema de processamento do sinal atrial otimizado. A experiência inicial desse sistema indica que a amplitude do sinal atrial em ritmo sinusal se mantém estável ao longo do tempo ${ }^{37}$, o que faz desse dispositivo como promissora alternativa ao CDI-DDD.

\section{Cardiodesfibrilador implantável transvenoso (CDI-TV) ou subcutâneo (CDI-S)}

O eletrodo subcutâneo de desfibrilação já vem sendo utilizado há mais de 20 anos em associação com eletrodos transvenosos ou epicárdicos ${ }^{38-41}$, em situações específicas (Fig. 4).

A solução encontrada atualmente para os problemas vasculares dos cabos-eletrodos nos pacientes apenas com

(a)

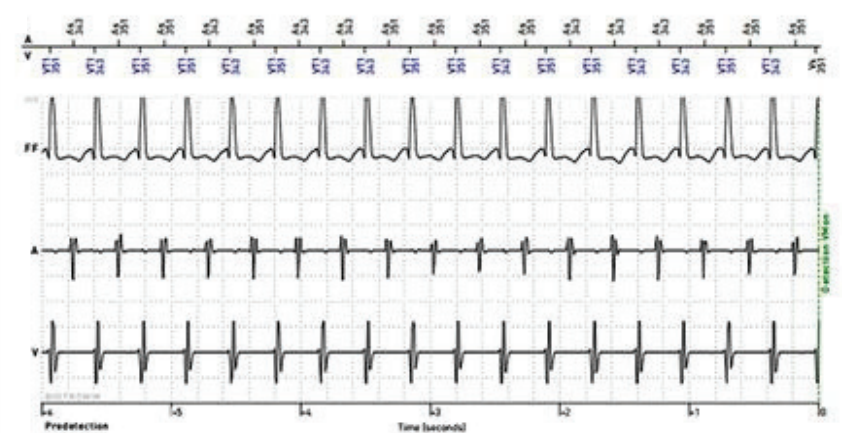

(b)

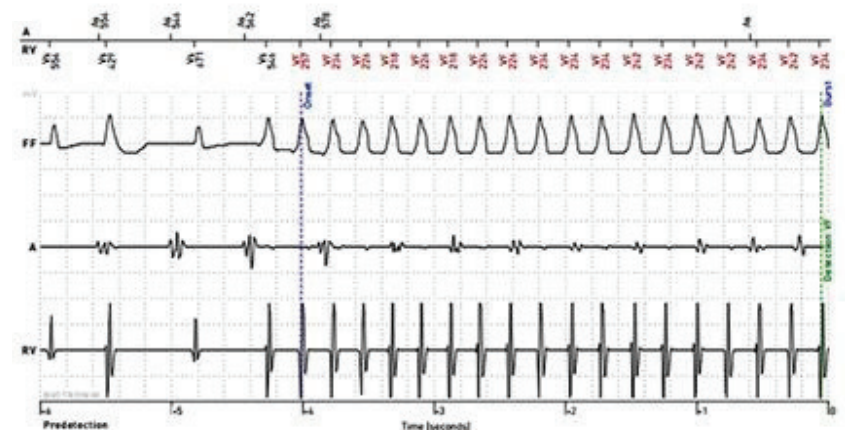

(c)

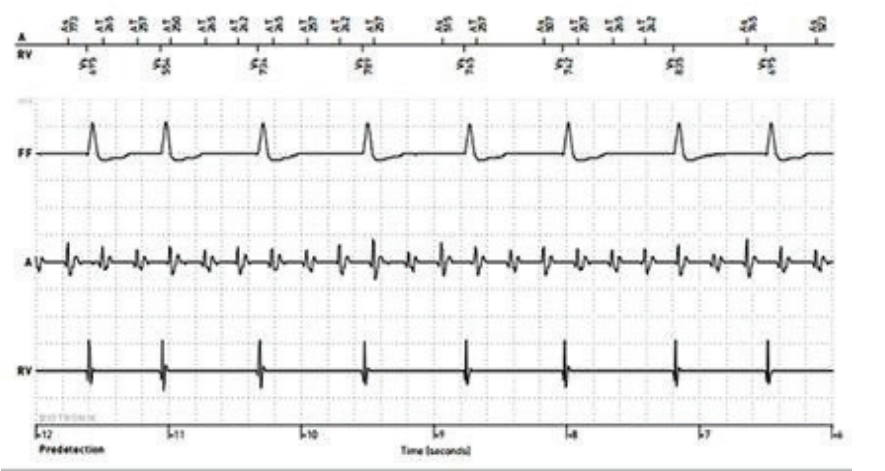

Figura 3. Exemplo de eletrogramas intracavitários durante (a) taquicardia supraventricular, (b) taquicardia ventricular e (c) flutter atrial. 
necessidade de prevenção de morte súbita e sem necessidade de estimulação cardíaca foi o desenvolvimento de um sistema de CDI inteiramente subcutâneo, onde o eletrodo de choque é colocado acima do esterno (Figs. 5 e 6).

Dois grandes estudos prospectivos demonstraram a eficácia e a segurança do CDI-S comparado ao CDI-TV (IDE [S-ICD System IDE Clinical Investigation] e EFFORTLESS [Boston Scientific Post Market S-ICD Registry]), em relação às complicações a curto e longo prazo, e à incidência de choques inapropriados.

O estudo EFFORTLESS ${ }^{43}$, que teve maior tempo de seguimento (5 anos), é um registro observacional, não randomizado, com mais de 800 pacientes arrolados em 42 centros clínicos, em 10 países. O objetivo do estudo foi demonstrar os desfechos a curto, médio e longo prazo do CDI-S. Foram excluídos do estudo pacientes com taquicardia ventricular (TV) espontânea, incessante ou recorrente, passível de tratamento por terapia
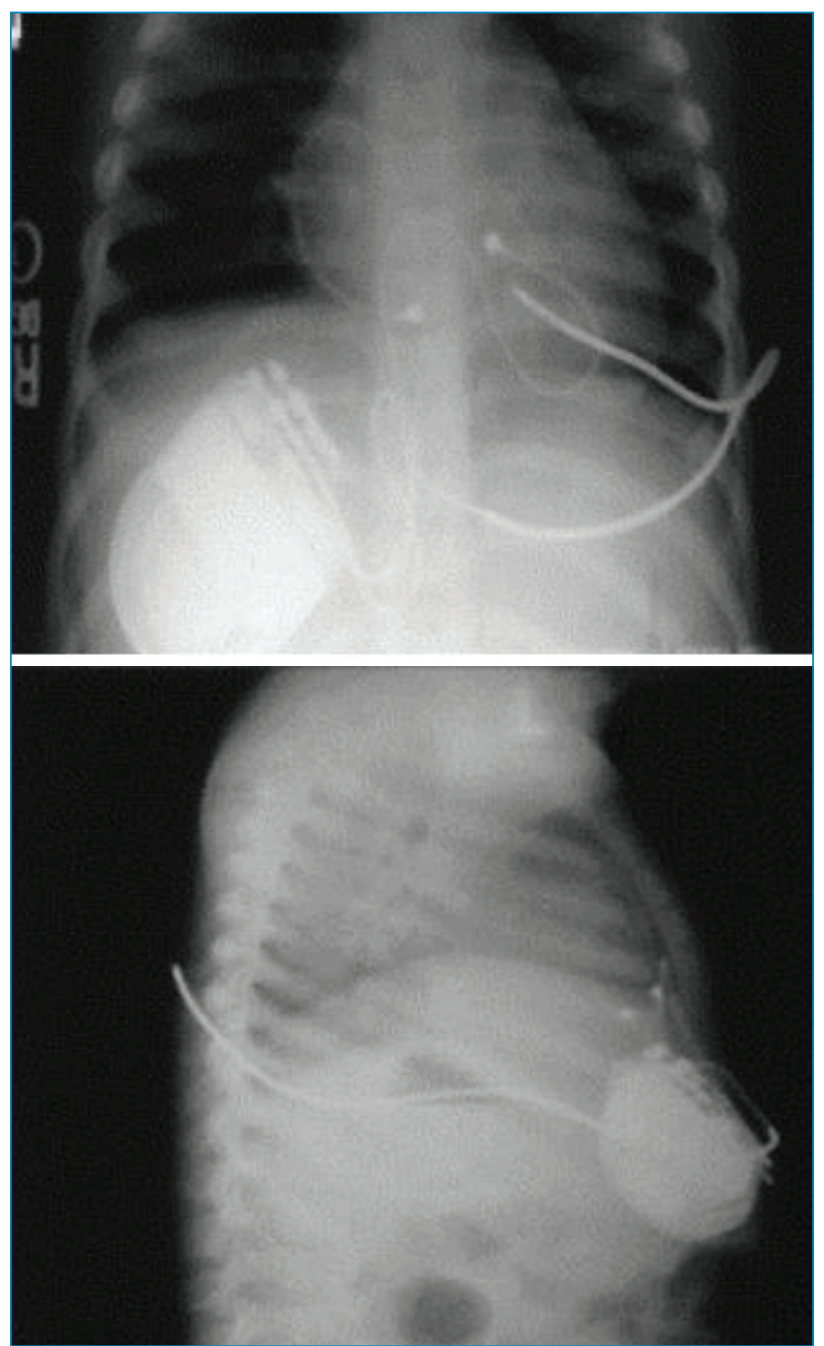

Figura 4. Radiografia de tórax demonstrando implante de eletrodo de desfibrilação subcutâneo (Medtronic Subcutaneous Lead Model 6996SQ) associado a eletrodo bipolar de estimulação/sensibilidade epicárdico em criança ${ }^{41}$. antitaquicardia (ATP); pacientes com indicação para terapia de ressincronização cardíaca (TRC) ou bradicardia sintomática, e pacientes portadores de marcapasso com estimulação unipolar. Esse estudo demonstrou eficácia de choque muito grande para arritmias ventriculares espontâneas e incidência reduzida de choques inapropriados. A taxa livre de complicações e a baixa taxa de mortalidade se estenderam além do primeiro ano de seguimento; assim como a taxa de choques inapropriados, risco de infecção e complicações gerais reduziram à medida que os médicos que realizam o procedimento ganharam mais experiência com o dispositivo ao longo do tempo ${ }^{44}$.

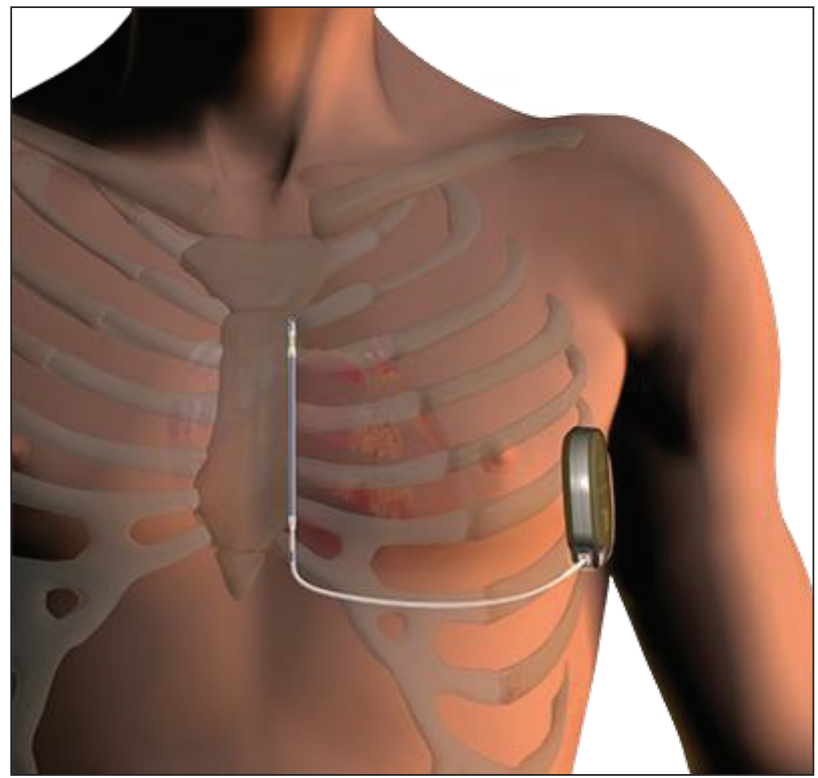

Figura 5. Representação gráfica do sistema de CDI-S da Boston Scientific - S-ICD SQRX ${ }^{42}$.
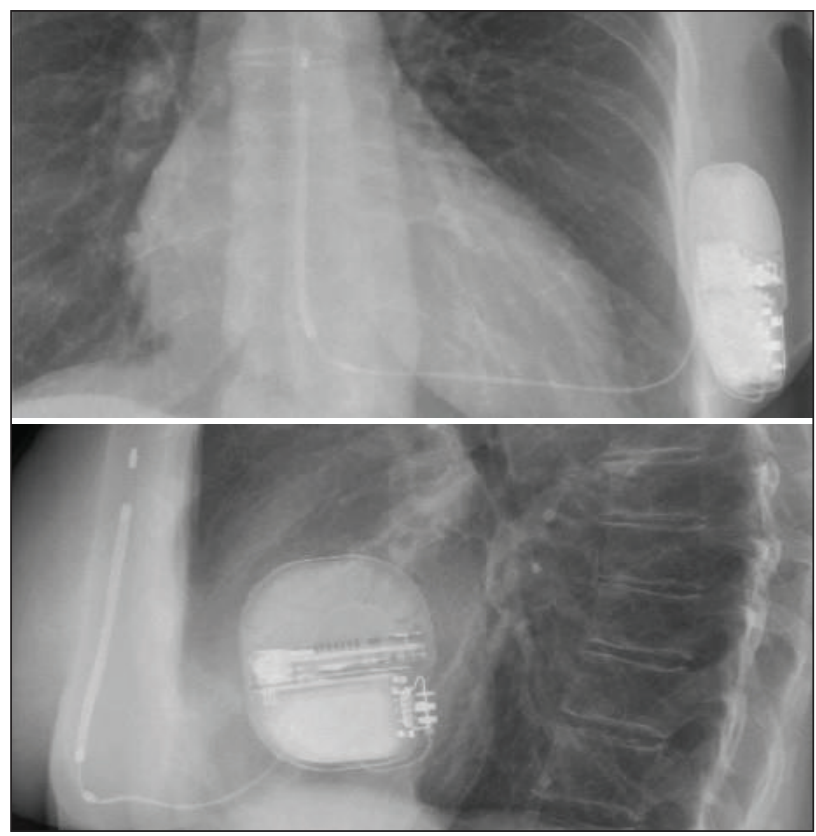

Figura 6. Radiografia pós-implante demonstrando a colocação ideal do gerador de pulsos e do eletrodo subcutâneo ${ }^{42}$. 
Desse modo, foi possível demonstrar a eficácia e segurança do CDI-S em pacientes com indicação de implante para prevenção primária e secundária, sem indicação para estimulação, em um período de acompanhamento superior a três anos ${ }^{43,45}$.

As últimas diretrizes da Sociedade Europeia de Cardiologia (SEC) já contemplam o CDI-S como opção terapêutica, com classe de recomendação IIa, para pacientes com indicação de CDI que não necessitem de estimulação para bradicardia, ressincronização cardíaca ou $\mathrm{ATP}^{46}$.

A escolha de CDI-TV para priorizar a ATP deve ser pautada em resultados de estudos como a subanálise do estudo SCD-HeFT ${ }^{47}$, em que $7 \%$ apresentaram mais de um episódio de TV durante seguimento de 46 meses, demonstrando o benefício anual de 1,8\% para o $\mathrm{ATP}^{48}$ e o Trial Painfree $\mathrm{Rx} \mathrm{II}^{49}$ que selecionou pacientes com substrato para TV monomórfica estável (excluindo pacientes em que se acredita ser improvável a ocorrência desse tipo de arritmia como, por exemplo, os portadores de miocardiopatia hipertrófica, síndrome do QT longo ou síndrome de Brugada), demonstrou uma redução de $42 \%$ de episódios de choque para TVs rápidas usando o ATP, em comparação à dispositivos programados somente com choque.

A associação de marcapassos sem eletrodos (leadless) em associação com o CDI-S para suprir essas deficiências fará com que os médicos não tenham que decidir entre a possibilidade de ATP e altas taxas de complicações dos eletrodos transvenosos.

\section{Marcapasso leadless (MPLL)}

Recentemente o MPLL surgiu como uma alternativa ao uso dos eletrodos transvenosos na estimulação cardíaca artificial e, apesar do curto tempo de experiência com esses dispositivos, eles têm se demonstrado seguros.

Atualmente dispomos de dois sistemas de estimulação leadless: (1) o Nanostim Leadless Cardiac Pacemaker (LCP; St. Jude Medical), e (2) o Micra Transcatheter Pacing System (TPS; Medtronic). Ambos dispositivos são unidades independentes (sem necessidade de gerador de pulso externo) aptas a realizar estimulação ventricular direita, detecção e resposta à frequência.

O primeiro ensaio a avaliar o uso do MPLL (LEADLESS Trial)50 foi um estudo multicêntrico, prospectivo, não randomizado, de braço único, que arrolou 33 pacientes, que foram submetidos a implante de MPLL entre dezembro de 2012 e abril de 2013, em três centros, com o intuito de avaliar a segurança e performance clínica desses dispositivos. $\mathrm{O}$ desfecho primário de segurança foi a ausência de complicações aos 90 dias. Os desfechos secundários incluíram a taxa de sucesso do implante, o tempo do implante e as medidas de desempenho do dispositivo (limiares de estimulação/ sensibilidade e performance de resposta à frequência).

A idade média da coorte de pacientes $(\mathrm{n}=33)$ foi de $77 \pm 8$ anos e $67 \%$ dos pacientes eram do sexo masculino $(\mathrm{n}=22 / 33)$. A indicação mais comum para estimulação cardíaca foi fibrilação atrial permanente com bloqueio atrioventricular $(\mathrm{n}=22,67 \%)$. A taxa de sucesso do implante foi de $97 \%(\mathrm{n}=32)$. Cinco pacientes $(15 \%)$ necessitaram do uso de > 1 MPLL durante o procedimento. Um paciente desenvolveu perfuração do ventrículo direito e tamponamento cardíaco durante o procedimento de implante e foi a óbito decorrente de acidente vascular cerebral. A taxa global livre de complicações foi de 94\% (31/33).

Após três meses de acompanhamento, as medidas de desempenho da estimulação (sensibilidade, impedância e limiar de estimulação) melhoraram ou foram estáveis dentro dos limites aceitos. Portanto, nessa experiência inicial, o MPLL de câmara única mostrou-se seguro e viável ${ }^{50}$.

O estudo LEADLESS II $^{51}$ foi um estudo prospectivo conduzido em 56 centros em três países (EUA, Canadá e Austrália), com 526 pacientes arrolados, com o objetivo de avaliar a segurança e eficácia do sistema de MPLL. A idade média dos pacientes foi de $75 \pm 8$ anos e $62 \%$ eram do sexo masculino. Do total de pacientes, 300 tiveram o tempo mínimo de seguimento de 6 meses. A taxa de sucesso do implante foi de 95,8\% (504/526), o tempo médio do procedimento foi de $28,6 \pm 17,8$ min e $70 \%$ dos pacientes não necessitaram de reposicionamento do dispositivo. Eventos adversos sérios ocorreram em 34 pacientes (6,5\%). Efusão pericárdica ocorreu em 1,5\% dos casos, porém somente 0,4\% necessitaram intervenção. Complicações vasculares ocorreram em 1,2\% e deslocamento do dispositivo em $1,1 \%$. Nas primeiras duas semanas após o implante, quatro dispositivos deslocaram para a artéria pulmonar e dois para a veia femoral direita. Todos foram removidos por via percutânea e novos MPLLs foram implantados. Além disso, $0,8 \%$ dos pacientes necessitaram de retirada do dispositivo devido aos limiares de estimulação elevados. Concluiu-se então que o MPLL atendeu aos requisitos pré-especificados de estimulação e detecção na grande maioria dos pacientes ${ }^{51}$.

Uma análise prospectiva multicêntrica publicada em $2016^{52}$ foi realizada para avaliar a eficácia e a segurança do 
Micra TPS. Foram arrolados 744 pacientes com indicações baseadas em diretrizes para estimulação ventricular em 56 centros em 19 países na América do Norte, Europa, Ásia, Austrália e África; 19 pacientes saíram do estudo por não consentirem ou por não apresentarem critérios de elegibilidade. O desfecho primário de segurança foi a ausência de complicações maiores: morte, disfunção permanente do dispositivo por problemas elétricos ou mecânicos, hospitalização, prolongação da hospitalização por pelo menos $48 \mathrm{~h}$ ou revisão do sistema. O desfecho primário de eficácia foi a manutenção de limiares de comando baixos e estáveis na visita de 6 meses após implante.

O implante do dispositivo foi realizado com sucesso em 99,2\% dos pacientes (719 em 725 indivíduos); dos 6 pacientes que não obtiveram sucesso no implante, 4 apresentaram complicações maiores: 3 com perfuração cardíaca e 1 com efusão pericárdica; 1 paciente apresentava anatomia venosa desfavorável, e 1 paciente em que não foi possível a obtenção de limiares de comando satisfatórios. Dos pacientes submetidos ao implante com sucesso, 98,3\% atenderam aos critérios pré-estabelecidos de limiar de captura aos 6 meses. Os critérios de segurança pré-especificados também foram atingidos e, apesar de ocorrerem 28 complicações maiores em 25 pacientes, 96\% dos pacientes não apresentaram tais complicações aos 6 meses $^{52}$.

Os dois primeiros sistemas de MPLL demonstraram desempenho semelhante e promessa inicial de eficácia e segurança. Ainda não há dados de desempenho de longo prazo dos sistemas leadless para determinar sua robustez tecnológica. À medida que a estimulação sem eletrodos evolui, tanto na tecnologia do dispositivo quanto na experiência do médico, é provável que as complicações relacionadas ao procedimento diminuam. Ensaios clínicos randomizados comparando dispositivos convencionais e leadless são necessários para determinar completamente as diferenças entre essas tecnologias na prática clínica.

\section{PERSPECTIVAS FUTURAS}

Para expandir os benefícios da estimulação sem eletrodos para mais pacientes, estão sendo feitos esforços para desenvolver sistemas leadless com comunicação multicomponente, capazes de executar estimulação bicameral, terapia de ressincronização cardíaca (TRC) ou servir com unidade para ATP nos casos de CDI-S.

Apesar de não ser um sistema exclusivamente autônomo de MPLL, outro sistema de estimulação vem

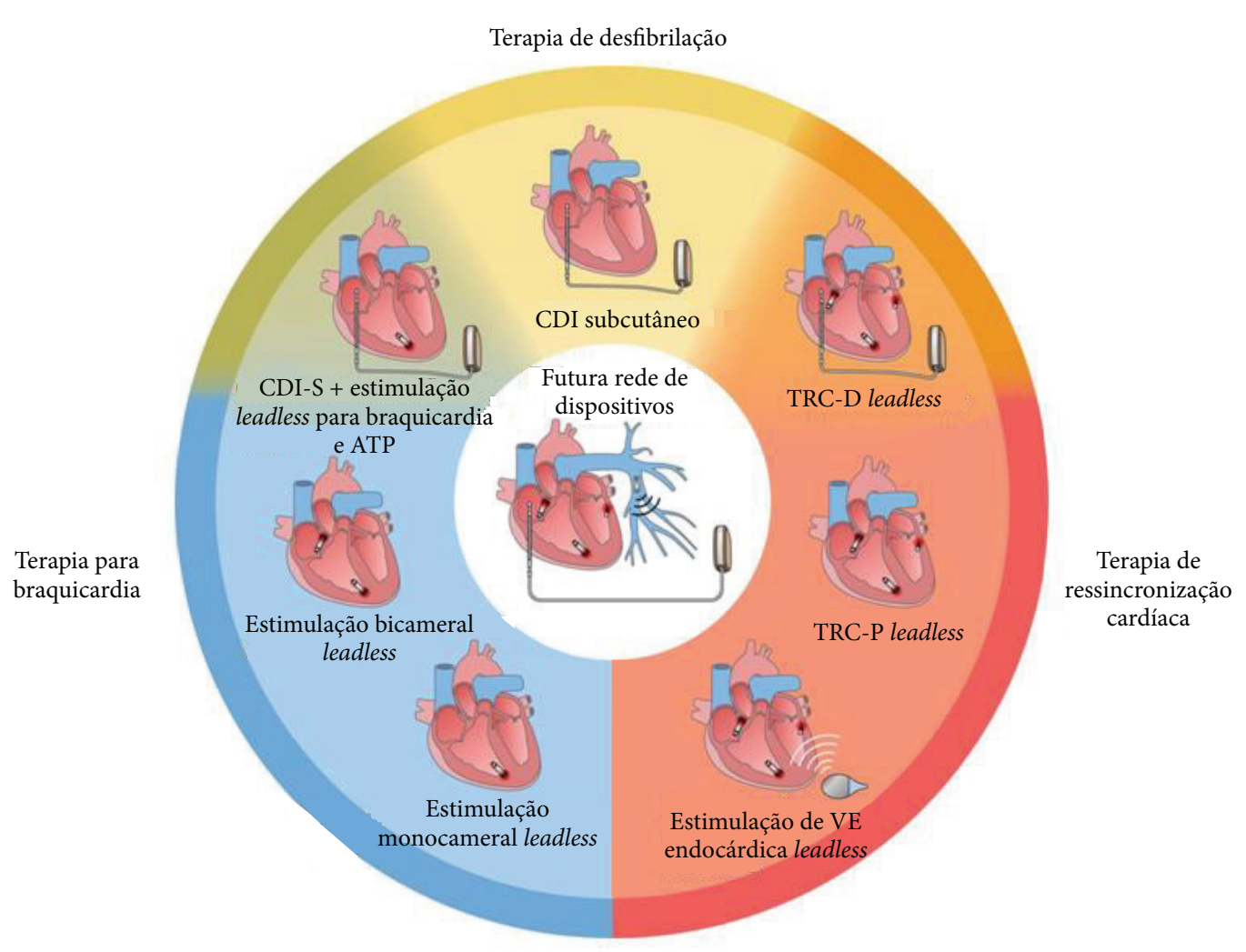

Figura 7. Perspectivas futuras para a estimulação cardíaca artificial ${ }^{56}$. 
sendo clinicamente investigado. Esse sistema consiste em dois componentes: (1) um eletrodo de estimulação sem cabo fixado na parede livre do ventrículo esquerdo e (2) um transmissor ultrassônico subcutâneo com uma bateria, que são sincronizados com o lado direito do sistema de estimulação e emite pulsos ultrassônicos. O eletrodo de estimulação converte a energia do ultrassom em estímulos elétricos, resultando em estimulação do ventrículo esquerdo para ressincronização cardíaca. Um grande estudo clínico multicêntrico da FDA está planejado para começar em um futuro próximo ${ }^{53}$.

Também é esperado que a estimulação sem eletrodos possa ser combinada com a terapia de desfibrilação. Apesar de não existir vasta experiência clínica com essa combinação, há relatos de implante concomitante, mas não comunicativo, de CDI-S e MPLL ${ }^{54,55}$.

Além disso, um terceiro sistema de MPLL que pode fornecer estimulação antitaquicardia (ATP) quando associado a um CDI-S foi testado com sucesso em estudo pré-clínico. Espera-se que os ensaios clínicos dessa terapia combinada comecem em breve ${ }^{56}$ (Fig. 7).

\section{CONCLUSÃO}

A estimulação cardíaca artificial segue em constante evolução desde as experiências iniciais na área, contudo ainda há grande preocupação em relação às complicações relacionadas aos implantes, principalmente às relacionadas aos cabos-eletrodos que ainda são responsáveis por morbidade significativa. Por esse motivo, deve-se sempre considerar a possibilidade de se implantar o mínimo possível de eletrodos transvenosos.

Há a expectativa de que nos próximos anos ocorram avanços tecnológicos e de conhecimento no campo da estimulação leadless, permitindo que esses dispositivos sejam incorporados na prática clínica de maneira rotineira.

Hoje, se o implante de eletrodos ventriculares nos casos de DNS com condução AV preservada for racionalizado, o implante de eletrodos atriais nos CDIs sem necessidade de estimulação antibradicardia ou dos eletrodos ventriculares nos casos sem a necessidade de ATP considerando o implante de CDIs subcutâneos, este artigo terá cumprido o seu papel.

\section{REFERÊNCIAS}

1. Raatikainen MJP, Arnar DO, Merkely B, Camm AJ, Hindricks G. Access to and clinical use of cardiac implantable electronic devices and interventional electrophysiological procedures in the European Society of Cardiology Countries: 2016 Report from the European Heart Rhythm Association. EP Eur. 2016; 18(IS 3):iii1-79. https://doi.org/10.1093/ europace/euw244

2. Greenspon AJ, Patel JD, Lau E, Ochoa JA, Frisch DR, Ho RT et al. Trends in permanent pacemaker implantation in the United States from 1993 to 2009: increasing complexity of patients and procedures. J Am Coll Cardiol. 2012; 60(16):1540-5. https://doi.org/10.1016/j.jacc.2012.07.017

3. Mond HG, Proclemer A. The 11th world survey of cardiac pacing and implantable cardioverter-defibrillators: calendar year 2009 - a World Society of Arrhythmia's Project. Pacing Clin Electrophysiol. 2011; 34(8):1013-27. https://doi. org/10.1111/j.1540-8159.2011.03150.x

4. Cohen TJ, Asheld WJ, Germano J, Islam S, Patel D. A comparative study of defibrillator leads at a large-volume implanting hospital: results from the Pacemaker and Implantable Defibrillator Leads Survival Study ("PAIDLESS"). J Invasive Cardiol. 2015; 27(6):292-300.

5. U.S. Food and Drug Administration. Safety: Medtronic Sprint Fidelis defibrillator leads. 2007. [citado em 31 Jul 2016].
Disponível em: https://www.accessdata.fda.gov/scripts/ cdrh/cfdocs/cfres/res.cfm?id=65383

6. U.S. Food and Drug Administration. Safety: St. Jude Medical, Riata and Riata ST silicone endocardial defibrillation leads: class 1 recall - failures with lead insulation. 2016. [citado em 31 Jul 2016]. Disponível em: https://www.accessdata.fda. gov/scripts/cdrh/cfdocs/cfres/res.cfm?id=105847

7. Kersten DJ, Yi J, Feldman AM, Brahmbhatt K, Asheld WJ, Germano J, et al. The number of recalled leads is highly predictive of lead failure: results from the Pacemaker and Implantable Defibrillator Leads Survival Study ("PAIDLESS"). J Invasive Cardiol. 2016; 17(4):E198-202.

8. Kirkfeldt RE, Johansen JB, Nohr EA, Jørgensen OD, Nielsen JC. Complications after cardiac implantable electronic device implantations: an analysis of a complete, nationwide cohort in Denmark. Eur Heart J. 2014; 35(18):1186-94. https://doi. org/10.1093/eurheartj/eht511

9. Udo EO, Zuithoff NP, van Hemel NM, de Cock CC, Hendriks T, Doevendans PA, Moons KG. Incidence and predictors of short and long-term complications in pacemaker therapy: The FOLLOWPACE study. Heart Rhythm. 2012; 9(5):728-35. https://doi.org/10.1093/europace/eus054

10. Kirkfeldt RE, Johansen JB, Nohr EA, Moller M, Arnsbo P, Nielsen JC. Pneumothorax in cardiac pacing: a population- 
based cohort study of 28860 Danish patients. EP Eur.2012; 14(8):1132-8. https://doi.org/10.1093/europace/eus054

11. Kiviniemi MS, Pirnes MA, Eränen HJK, Kettunen RVJ, Hartikainen JEK. Complications related to permanent pacemaker therapy. Pacing Clin Electrophysiol. 1999; 22(5):711-20. https://doi.org/10.1111/j.1540-8159.1999. tb00534.x

12. Ellenbogen KA, Hellkamp AS, Wilkoff BL, Camunãs JL, Love JC, Hadjis TA, et al. Complications arising after implantation of DDD pacemakers: the MOST experience. Am J Cardiol. 2003; 92(6):740-1. https://doi.org/10.1016/S00029149(03)00844-0

13. Haghjoo M, Nikoo MH, Fazelifar AF, Alizadeh A, Emkanjoo Z, Sadr-Ameli MA. Predictors of venous obstruction following pacemaker or implantable cardioverter-defibrillator implantation: a contrast venographic study on 100 patients admitted for generator change, lead revision, or device upgrade. EP Eur. 2007; 9(5):328-32. https://doi.org/10.1093/ europace/eum019

14. Harcombe AA, Newell SA, Ludman PF, WistowTE, Sharples LD, Schofield PM, et al. Late complications following permanent pacemaker implantation or elective unit replacement. Heart. 1998; 80(3):240-4. https://doi.org/10.1136/hrt.80.3.240

15. Brunner MP, Cronin EM, Wazni O, Baranowski B, Saliba WI, Sabik JF, et al. Outcomes of patients requiring emergent surgical or endovascular intervention for catastrophic complications during transvenous lead extraction. Heart Rhythm. 2014; 11(3):419-25. https://doi.org/10.1016/j. hrthm.2013.12.004

16. Tarakji KG, Wazni OM, Harb S, Hsu A, Saliba W, Wilkoff BL. Risk factors for 1-year mortality among patients with cardiac implantable electronic device infection undergoing transvenous lead extraction: the impact of the infection type and the presence of vegetation on survival. EP Eur. 2014; 16(10):1490-5. https://doi.org/10.1093/europace/euu147

17. Ahmed FZ, Fullwood C, Zaman M, Qamruddin A, Cunnington C, Mamas MA, et al. Cardiac implantable electronic device (CIED) infections are expensive and associated with prolonged hospitalisation: UK retrospective observational study. PLOS ONE. 2019; 14(3):1-13. https://doi.org/10.1371/ journal.pone.0213682

18. Hauser RG, Hayes DL, Kallinen LM, Cannom DS, Epstein AE, Almquist AK, et al. Clinical experience with pacemaker pulse generators and transvenous leads: An 8-year prospective multicenter study. Heart Rhythm. 2007; 4(2):154-60. https:// doi.org/10.1016/j.hrthm.2006.10.00919

19. Maisel WH, Hauser RG, Hammill SC, Hauser RG, Ellenbogen $K A$, Epstein $A E$, et al. Recommendations from the Heart Rhythm Society Task Force on Lead Performance Policies and Guidelines. Heart Rhythm. 2009; 6(6):869-85. https:// doi.org/10.1016/j.hrthm.2009.04.02420

20. Connolly SJ, Kerr CR, Gent M, Roberts RS, Yusuf S, Gillis AM, et al. Effects of Physiologic Pacing versus Ventricular Pacing on the Risk of Stroke and Death Due to Cardiovascular
Causes. N Engl J Med. 2000; 342(19):1385-91. https://doi. org/10.1056/NEJM20000511342190221

21. Kerr CR, Connolly SJ, Abdollah H, Roberts RS, Gent M, Yusuf $S$, et al. Canadian trial of physiological pacing: effects of physiological pacing during long-term follow-up. Circulation. 2004; 109(3):357-62. https://doi.org/10.1161/01. CIR.0000109490.72104.EE22

22. Lamas GA, Lee $\mathrm{KL}$, Sweeney MO, Silverman R, Leon A, Yee R. Ventricular pacing or dual-chamber pacing for sinus-node dysfunction. N Engl J Med. 2002; 346(24):1854-62. https:// doi.org/10.1056/NEJMoa01304023

23. Healey JS, Toff WD, Lamas GA, Andersen HR, Thorpe $K E$, Ellenbogen KA, et al. Cardiovascular outcomes with atrialbased pacing compared with ventricular pacing metaanalysis of randomized trials, using individual patient data. Circulation. 2006; 114(1):11-7. https://doi.org/10.1161/ CIRCULATIONAHA.105.610303

24. Castelnuovo E, Stein K, Pitt M, Garside R, Payne L. The effectiveness and cost-effectiveness of dual-chamber pacemakers compared with single-chamber pacemakers for bradycardia due to atrioventricular block or sick sinus syndrome: systematic review and economic evaluation. Health Technol Assess. 2005; 9(43):1-269. https://doi. org/10.3310/hta943025

25. Nielsen JC, Thomsen PEB, Højberg S, Møller M, Vesterlund T, Dalsgaard D, et al. A comparison of single-lead atrial pacing with dual-chamber pacing in sick sinus syndrome. Eur Heart J.2011; 32(6):686-96. https://doi.org/10.1093/eurheartj/ ehr02226

26. Senaratne J, Herath T, Beaudette D, Irwin M, Gulamhusein S, Senaratne M. Safety and efficacy of AAIR pacing in selected patients with sick sinus syndrome. Medicine. 2018; 97(42):16. https://doi.org/10.1097/MD.000000000001283327

27. Fuganti CJ, Melo CS, Moraes Junior AV, Pachon-Mateos JC, Pereira WL, Galvão Filho SS, et al. Diretrizes Brasileiras de Dispositivos Cardíacos Eletrônicos Implantáveis do Departamento de Estimulação Cardíaca Artificial (DECA) da Sociedade Brasileira de Cirurgia Cardiovascular (SBCCV). J Cardiac Arrythmias. 2015; 28(2 Supl):S1-6228.

28. Brignole M, Auricchio A, Baron-Esquivias, G, Bordachar, $\mathrm{P}$, Boriani G, Breithardt O-A, et al. 2013 ESC Guidelines on cardiac pacing and cardiac resynchronization therapy: The Task Force on cardiac pacing and resynchronization therapy of the European Society of Cardiology (ESC). Developed in collaboration with the European Heart Rhythm Association (EHRA). Eur Heart J. 2013; 34(29):2281-329. https://doi. org/10.1093/eurheartj/eht15029

29. Kusumoto FM, Schoenfeld MH, Barrett C, Edgerton JR, Ellenbogen KA, Gold MR, et al. 2018 ACC/AHA/HRS Guideline on the evaluation and management of patients with bradycardia and cardiac conduction delay: a report of the American College of Cardiology/American Heart Association Task Force on Clinical Practice Guidelines and the Heart Rhythm Society. Circulation. 2018; 140(8):e382-482. https:// doi.org/10.1161/CIR.000000000000062830 
30. Rattanawong P, Kewcharoen J, Mekraksakit P, Mekritthikrai R, Prasitlumkum N, Vutthikraivit W, et al. Device infections in implantable cardioverter defibrillators versus permanent pacemakers: A systematic review and meta-analysis. J Cardiovasc Electrophysiol. 2019; 30(7):1053-65. https://doi. org/10.1111/jce.13932

31. Kleemann $T$, Becker $T$, Doenges $K$, Vater $M$, Senges J, Schneider S, et al. Annual rate of transvenous defibrillation lead defects in implantable cardioverter-defibrillators over a period of $>10$ years. Circulation. 2007; 115(19):2474-80. https://doi.org/10.1161/CIRCULATIONAHA.106.663807

32. Dewland TA, Pellegrini CN, Wang Y, Marcus GM, Keung E, Varosy PD. Dual-chamber implantable cardioverterdefibrillator selection is associated with increased complication rates and mortality among patients enrolled in the NCDR implantable cardioverter-defibrillator registry. J Am Coll Cardiol. 2011; 58(10):1007-13. https://doi. org/10.1016/j.jacc.2011.04.03933

33. Konstantino $Y$, Haim M, Boxer J, Goldenberg I, Feldman A, Michowitz $Y$, et al. Clinical outcomes of single- versus dual-chamber implantable cardioverter defibrillators: lessons from the Israeli ICD registry. J Cardiovasc Electrophysiol.2016; 27(6):718-23. https://doi.org/10.1007/ s00392-019-01584-×34

34. 34. Bogossian H, Frommeyer G, Hochadel M, Ince H, Spitzer SG, Eckardt L, et al. Single chamber implantable cardioverter defibrillator compared to dual chamber implantable cardioverter defibrillator: less is more! Data from the German Device Registry. Clin Res Cardiol. 2019. https://doi. org/10.1007/s00392-019-01584-×35

35. Defaye P, Boveda S, Klug D, Beganton F, Piot O, Narayanan $K$, et al. (2017). Dual- vs. single-chamber defibrillators for primary prevention of sudden cardiac death: long-term follow-up of the Défibrillateur Automatique Implantable Prévention primaire registry. EP Eur.2017; 19(9):1478-84. https://doi.org/10.1093/europace/euw23036

36. Auricchio A, Hudnall JH, Schloss EJ, Sterns LD, Kurita $T$, Meijer A, et al. Inappropriate shocks in single-chamber and subcutaneous implantable cardioverter-defibrillators: a systematic review and meta-analysis. EP Eur. 2017;19(12):1973-80. https://doi.org/10.1093/europace/ euw41537

37. Worden NE, Alqasrawi M, Krothapalli SM, Mazur A. "Two for the price of one": A single-lead implantable cardioverterdefibrillator system with a floating atrial dipole. J Atr Fibrillation. 2016; 8(6):1396.38.

38. Higgins SL, Alexander DC, Kuypers CJ, Brewster SA. The subcutaneous array: a new lead adjunct for the transvenous ICD to lower defibrillation thresholds. Pacing Clin Electrophysiol. 1995; 18(8):1540-8. https://doi. org/10.1111/j.1540-8159.1995.tb06740.x

39. Kühlkamp V, Dörnberger $\vee$, Khalighi K, Suchalla CMR, Ziemer $G$, Seipel L. Effect of a single element subcutaneous array electrode added to a transvenous electrode configuration on the defibrillation field and the defibrillation threshold.
Pacing Clin Electrophysiol. 1998; 21(12):2596-605. https:// doi.org/10.1111/j.1540-8159.1998.tb00036.x

40. Gradaus R, Block M, Seidl K, Brunn J, Isgro F, Hammel D, et al. Defibrillation efficacy comparing a subcutaneous array electrode versus an "active can" implantable cardioverter defibrillator and a subcutaneous array electrode in addition to an "active can" implantable cardioverter defibrillator: results from active can versus array trials I and II.J Cardiovasc Electrophysiol. 2001; 12(8):921-7. https://doi.org/10.1046/ j.1540-8167.2001.00921.x

41. Madan N, Gaynor JW, Tanel R, Cohen, M, Nicholson S, Vetter $\checkmark$, Rhodes L. Single-finger subcutaneous defibrillation lead and "active can": a novel minimally invasive defibrillation configuration for implantable cardioverter-defibrillator implantation in a young child. J Thorac Cardiovasc Surg. 2003; 126(5):1657-9. https://doi.org/10.1016/S00225223(03)01032-8

42. Boston Scientific. Produtos - Desfibriladores (CDI) - Sistema S-ICD ${ }^{\text {TM }}$ C2020. [citado em: 3 Abr 2020]. Disponível em: https://www.bostonscientific.com/pt-BR/products/ defibrillators/s-icd1.html

43. Lambiase PD, Barr C, Theuns DAMJ, Knops R, Neuzil P, Johansen JB, et al. Worldwide experience with a totally subcutaneous implantable defibrillator: early results from the EFFORTLESS S-ICD Registry. Eur Heart J. 2014; 35(25):1657-65. https://doi.org/10.1093/eurheartj/ehu112

44. Knops RE, Nordkamp LRAO, GrootJR, Wilde AAM. Two-incision technique for implantation of the subcutaneous implantable cardioverter-defibrillator. Heart Rhythm. 2013; 10(8):124043. https://doi.org/10.1016/j.hrthm.2013.05.01645

45. Weiss R, Knight BP, Gold MR, Leon AR, Herre JM, Hood M, et al. Safety and efficacy of a totally subcutaneous implantablecardioverter defibrillator. Circulation. 2013; 128(9):944-53. https://doi.org/10.1161/CIRCULATIONAHA.113.003042

46. Priori SG, Blomström-Lundqvist C, Mazzanti A, Blom N, Borggrefe M, Camm J, et al. 2015 ESC Guidelines for the management of patients with ventricular arrhythmias and the prevention of sudden cardiac death. Rev Esp Cardiol (English Edition). 2016; 69(2):176. https://doi.org/10.1016/j. rec.2016.01.001

47. Bardy $\mathrm{GH}$, Lee $\mathrm{KL}$, Mark DB, Poole JE, Packer $\mathrm{DL}$, Boineau $\mathrm{R}$, et al. Amiodarone or an implantable cardioverterdefibrillator for congestive heart failure. N Engl J Med. 2005; 352(3):225-37. http://doi.org/10.1056/nejmoa043399

48. Poole JE, Gold MR. Who should receive the subcutaneous implanted defibrillator? The subcutaneous implantable cardioverter defibrillator (ICD) should be considered in all ICD patients who do not require pacing. Circulation. 2013; 6(6):1236-45. https://doi.org/10.1161/CIRCEP.113.000481

49. Wathen MS, DeGroot PJ, Sweeney MO, Stark AJ, Otterness MF, Adkisson WO, et al. Prospective randomized multicenter trial of empirical antitachycardia pacing versus shocks for spontaneous rapid ventricular tachycardia in patients with implantable cardioverter-defibrillators: Pacing Fast Ventricular Tachycardia Reduces Shock Therapies (PainFREE 
Rx II) trial results. Circulation. 2004; 110(17):2591-6. https:// doi.org/10.1161/01.CIR.0000145610.64014.E4

50. 50. Reddy VY, Knops RE, Sperzel J, Miller MA, Petru J, Simon J, et al. Permanent leadless cardiac pacing: results of the LEADLESS trial. Circulation. 2014; 129(14):1466-71. https:// doi.org/10.1161/CIRCULATIONAHA.113.006987

51. 51. Reddy VY, Exner DV, Cantillon DJ, Doshi R, Bunch TJ, Tomassoni GF, et al. Percutaneous implantation of an entirely intracardiac leadless pacemaker. N Engl J Med. 2015; 373:1125-35. https://doi.org/10.1056/NEJMoa1507192

52. 52. Reynolds D, Duray GZ, Omar R, Soejima K, Neuzil P, Zhang $\mathrm{S}$, et al. A leadless intracardiac transcatheter pacing system. N Engl J Med. 2016; 374(6):533-41. https://doi. org/10.1056/NEJMoa151164353

53. 53. Auricchio A, Delnoy P-P, Butter C, Brachmann J, Van Erven L, Spitzer S, et al. Feasibility, safety, and short-term outcome of leadless ultrasound-based endocardial left ventricular resynchronization in heart failure patients: results of the
Wireless Stimulation Endocardially for CRT (WiSE-CRT) study. EP Eur. 2014; 16(5):681-8. https://doi.org/10.1093/ europace/eut43554

54. 54. Tjong FVY, Brouwer TF, Smeding L, Kooiman KM, Groot $J R$, Ligon D, et al. Combined leadless pacemaker and subcutaneous implantable defibrillator therapy: feasibility, safety, and performance. EP Eur. 2016; 18(11):1740-7. https://doi.org/10.1093/europace/euv45755

55. 55. Mondésert B, Dubuc M, Khairy P, Guerra PG, Gosselin G, Thibault B. Combination of a leadless pacemaker and subcutaneous defibrillator: first in-human report. Heart Rhythm Case Rep. 2015; 1(6):469-71. https://doi. org/10.1016/j.hrcr.2015.07.00956

56. 56. Tjong FVY, Brouwer TF, Kooiman KM, Smeding L, Koop $B$, Soltis $B$, et al. Communicating antitachycardia pacingenabled leadless pacemaker and subcutaneous implantable defibrillator. J Am Coll Cardiol. 2016; 67(15):1865-66. https:// doi.org/10.1016/j.jacc.2016.02.039 